\title{
Crenças de autoeficácia e resiliência: apontamentos da literatura sociocognitiva
}

\author{
Self-efficacy beliefs and resilience: findings \\ of social-cognitive literature
}

\author{
Arlete Portella FONTES \\ Roberta Gurgel AZZI
}

\begin{abstract}
Resumo
Este artigo investiga como as relações entre autoeficácia e resiliência vêm sendo abordadas pela literatura em diferentes etapas do ciclo de vida e contextos diversos. Toma como referência a teoria social cognitiva de Bandura e aborda os conceitos de agência, autoeficácia e autorregulação. As crenças de autoeficácia são tidas como percepções sobre a capacidade de organizar e executar determinado curso de ação; são consideradas elemento central na fundação da motivação e da realização e podem afetar a maneira como o indivíduo resiste às pressões do ambiente, sem entrar em disfuncionalidade (resiliência). Enquanto agente, o indivíduo encara as ameaças do ambiente como oportunidades, escolhendo estratégias para lidar com elas. Por meio de mecanismos autorregulatórios, os indivíduos motivam-se, orientam-se por meio do controle proativo, estabelecendo metas desafiadoras e mobilizando esforços para alcançar seus objetivos. Estudos baseados na crença de autoeficácia e resiliência são destacados e alguns horizontes são relacionados para pesquisa.
\end{abstract}

Unitermos: Agência. Autoeficácia. Autorregulação. Resiliência.

\begin{abstract}
This article studies how the relationship between self-efficacy and resilience has been dealt with by literature in different phases of the life cycle and contexts. It takes Bandura's Social Cognitive Theory as a reference, addressing the concepts of agency, self-efficacy and self-regulation. Self-efficacy beliefs are seen as perceptions about the capacity to organize and execute a specific course of action; they are regarded as a central element in the foundation of motivation and attainment and may affect the manner in which individuals cope with taxing environmental demands without falling into dysfunction (resilience). As an agent, the individual sees environmental threats as opportunities, choosing strategies to deal with them. Using self-regulating mechanisms, the individuals motivate themselves and steer themselves through proactive control, establishing challenging goals and mobilizing efforts to achieve the desired goals. Studies referring to the beliefs of self-efficacy and resilience are highlighted, and some horizons are compared for research.
\end{abstract}

Uniterms: Agency. Self-efficacy. Self-regulation. Resilience.

$\boldsymbol{\nabla \nabla \nabla \nabla}$

1 Universidade Estadual de Campinas, Faculdade Ciências Médicas, Pós-Graduação em Gerontologia. Campinas, SP, Brasil.

2 Universidade Estadual de Campinas, Faculdade de Educação, Departamento de Psicologia Educacional. Av. Bertrand Russel, 81, Barão Geraldo, 13084-100, Campinas, SP, Brasil. Correspondência para/Correspondence to: A.P. FONTES. E-mail: <arletepfontes@gmail.com>. 
Este artigo tem como objetivo mostrar, em linhas gerais, como a teoria social cognitiva aborda as relações entre autoeficácia e resiliência em diferentes contextos e etapas do curso de vida, e também revelar algumas evidências de pesquisa em que tais relações vêm sendo investigadas.

As crenças de autoeficácia, como crenças que os indivíduos têm em sua própria capacidade de organizar e executar determinado curso de ação para alcançar determinado resultado (Bandura,1977; 1997), têm sido estudadas em diferentes etapas do curso de vida (adolescência, adulto, velhice) e diferentes contextos (recuperação pós-trauma, organizações, escolas). A resiliência é definida como a capacidade de recuperação e manutenção do comportamento adaptativo, quando ameaçado por um evento estressante, segundo Staudinger, Marsiske e Baltes (1995). Para melhor compreensão da relação entre as crenças de autoeficácia e resiliência, apoiou-se nos conceitos de agência, autorregulação e crenças de autoeficácia, desenvolvidos pela teoria social cognitiva, proposta por Bandura (1986).

Bandura (2001) utiliza-se do conceito de agência para explicar o funcionamento humano e os processos de autodesenvolvimento, adaptação e mudança nele envolvidos. Como agente, o indivíduo influencia o próprio comportamento e as circunstâncias de sua vida, pois não é apenas alvo das influências do meio, mas também age sobre ele e produz influências que o modificam.

Ao descrever o exercício da agência humana, Bandura (2008) refere-se a características humanas básicas: a intencionalidade, como a capacidade de fazer planos e visualizar estratégias de ação para realizá-los; a antecipação, por meio da qual o indivíduo cria objetivos para si mesmo, prevê os resultados prováveis de seus atos, guia e motiva seus esforços; a autorregulação, que Ihe permite adotar padrões de referência para avaliar, monitorar e regular seus atos e, finalmente, a reflexão, capacidade através da qual o indivíduo reflete sobre o valor e o significado de suas ações, fazendo ajustes, quando necessário.

A autorregulação pode ser entendida como a capacidade de o organismo produzir discrepâncias em relação a um padrão esperado e de criar um sistema reativo que possibilite a redução dessas discrepâncias (Bandura, 2006). Através de mecanismos autorregula- dores, os indivíduos motivam-se, orientam-se por meio do controle proativo, estabelecendo metas desafiadoras e padrões de desempenho difíceis para si mesmos; mobilizam seus esforços para alcançar os objetivos desejados; exercem automonitoramento, monitoramento do tempo e autoavaliação; e refletem sobre o valor e o significado. À medida que vão comparando seus resultados com o padrão desejado, entra em ação o controle reativo das discrepâncias. O processo autorregulatório opera sobre diversas áreas do funcionamento humano: na saúde, na regulação do afeto, no desempenho nas organizações, nos esportes, na educação e na cultura (Bandura, 1997; Bandura \&Wood, 1989).

Ainda, dentre os mecanismos de agência pessoal, nenhum deles é mais central ou determinante do que as crenças na capacidade de exercer controle sobre o meio e as situações vividas. As crenças de autoeficácia têm papel crucial para a determinação do comportamento humano (Bandura, 1997).

Bandura (2004) propõe quatro mecanismos para desenvolvimento de um forte senso de autoeficácia: 1) modelação social: a observação de outros atuando como modelos bem-sucedidos permite que o indivíduo identifique conhecimentos e habilidades para lidar com as exigências do meio; 2) persuasão social: quando as pessoas são persuadidas a atuar de forma a obter sucesso, elas confiam mais em sua capacidade e afastamse de situações nas quais podem falhar; além disso, não se envolvem em pensamentos que intensificam as dúvidas sobre sua própria capacidade; 3) estados emocionais: as pessoas também julgam suas capacidades em função de seus estados emocionais, fazendo leituras de sua tensão, ansiedade e depressão como sinais de sua deficiência pessoal; e, finalmente, 4) experiências de maestria, ou seja, as experiências de domínio em relação ao exercício de alguma atividade ou habilidade apresentadas como o mais efetivo recurso para fortalecer crenças de autoeficácia.

Há especial interesse em conhecer como tais crenças poderiam atuar como facilitadores e atenuantes das diversas pressões proporcionadas pelo meio ambiente, algumas delas hostis e desvantajosas para a adaptação, como as mudanças púberes na adolescência ou a redução de reservas, própria do envelhecimento, a combinação de fatores econômicos e sociais (pobreza, 
exclusão social), os eventos de vida desvantajosos (morte dos pais, alcoolismo, perdas financeiras), até os estressores advindos de situações traumáticas (catástrofes ambientais, atentados, sequestros).

A maneira como os indivíduos enfrentam tais pressões e estressores no transcorrer do curso de vida vai determinar sua capacidade de adaptação e recuperação; em outras palavras, sua resiliência. Bandura (2002) destaca que o bem-estar e as realizações humanas requerem senso de eficácia resiliente, pois o cotidiano interpõe vários obstáculos ao indivíduo.

Entre os obstáculos do cotidiano, do ponto de vista de enfrentamento de condições adversas, Barreira e Nakamura (2006) sugerem, inspirados em Bandura (1997), que se estudem os conceitos de resiliência e autoeficácia como relacionados ao enfrentamento das mudanças sociais, econômicas e laborais trazidas pela globalização, envolvendo contexto, cultura e responsabilidade coletiva.

O termo resiliência, utilizado na física para indicar uma característica de materiais que conseguem retornar a sua forma original depois de terem sido submetidos a pressões, foi incorporado pela psicologia para designar a capacidade do indivíduo de adaptar-se de maneira positiva às situações adversas, mantendo seu desenvolvimento normal e sendo capaz de recuperar-se dos efeitos estressores (Lopes, 2005).

Historicamente, os estudos sobre resiliência foram efetuados pela psicopatologia e pelos estudos do comportamento infantil e adolescente. Recentemente, esse conceito vem sendo investigado nos mais diversos contextos: no ambiente acadêmico, incluindo professores e alunos; nas diferentes etapas do curso de vida (adolescência, vida adulta e velhice); na recuperação do indivíduo após situações traumáticas; e no desempenho nas organizações (Bandura, 1997). Diante de cada um desses contextos, o indivíduo pode encarar as pressões como ameaça ou como uma oportunidade, exercendo seu papel de agente e concentrando-se nas possibilidades que as situações proporcionam, em função de suas crenças sobre sua capacidade de atuar como agente sobre elas (Bandura, 2008).

O conceito de resiliência, do ponto de vista da teoria social cognitiva, repousa basicamente sobre três pilares: a capacidade de o indivíduo atuar como agente diante das condições adversas, persistindo em seus esforços e voltar à normalidade; a capacidade de regular seu comportamento, apoiando-se em seus resultados, quando comparados a um padrão de referência; e, principalmente, a capacidade de acreditar em sua competência para fazê-lo.

\section{Método}

Realizou-se inicialmente uma busca geral de artigos pelas palavras-chave self-efficacy e resilience, em textos sobre a teoria social cognitiva, arquivados em banco de dados do Núcleo de Estudos Avançados sobre Psicologia Comportamental e Cognitiva (Neapsi) da Faculdade de Educação da Unicamp (Quadro 1). Em seguida, promoveu-se uma busca sistematizada na base de dados Medline, Scielo, e PsychINFO, no período de 2003 a 2008 (de março a julho), excluindo os artigos referentes à teoria social cognitiva (Quadro 2).

Foi encontrado um total de 21 artigos e capítulos de livros, 13 deles referentes à teoria social cognitiva (Quadro 1), os quais permitiram a organização de núcleos temáticos: crenças de autoeficácia e resiliência na adolescência, no desenvolvimento ao longo da vida, na recuperação pós-trauma, em professores, na vida acadêmica e em organizações.

Os outros 8 artigos encontrados foram distribuídos entre as categorias previamente definidas pelas autoras, com base na pesquisa anterior sobre a teoria social cognitiva, conforme indicado pela (Quadro 2). Acrescentou-se à categorização original a categoria: desafios de pesquisa. Os artigos foram analisados buscando evidências de relações entre os conceitos de autoeficácia e resiliência. O artigo nacional de Barreira e Nakamura (2006) - "Autoeficácia percebida e resiliência: articulações entre conceitos" - foi abordado na introdução deste texto, não tendo sido contemplado em nenhuma das categorias descritas acima por estar sua contribuição direcionada para foco diverso do deste trabalho.

A seguir, são discutidas as relações entre autoeficácia e resiliência em diferentes contextos e etapas do curso de vida, encontradas nos artigos localizados. 
Quadro 1. Relação de artigos e capítulos de livros referentes à teoria social cognitiva

\begin{tabular}{lll}
\hline Artigos/capítulos de livros & Autores & Ano \\
\hline 1. Self-efficacy: toward a unifying theory of behavioral change & Bandura & 1977 \\
2. Social foundations of thought and action: a social cognitive theory & Bandura & 1986 \\
3. Effect of perceived controllability and performance standards on self-regulation & Bandura \& Wood & 1989 \\
of complex decision making & Bandura & 1997 \\
4. Self-efficacy: the exercise of control & Bandura & 2001 \\
5. Social cognitive theory: an agentic perspective & Zimmerman & 2002 \\
6. Achieving self-regulation. The trial and triumph of adolescence & 2002 \\
7. Environmental sustainability by sociocognitive deceleration of population & Bandura & \\
growth & & 2004 \\
8. Swimming against the mainstream: the early years from chilly tributary to & Bandura & \\
transformative mainstream & & 2004 \\
9. Social cognitive theory of posttraumatic recovery: the role of perceived self-efficacy & Benight \& Bandura & 2005 \\
10. Impact of adolescents' filial self-efficacy on quality of family functioning and & Caprara, Pastorelli, Regalia, Scabini \& Bandura & 2006 \\
satisfaction & Azzi, Polydoro \& Bzuneck & 2006 \\
11. Considerações sobre a autoeficácia docente & Bandura \\
12. Towards a psychology of human agency & Bandura \\
13. A teoria social cognitiva na perspectiva da agência. & 2008 \\
\hline
\end{tabular}

Fonte: Núcleo de Estudos Avançados sobre Psicologia Comportamental e Cognitiva (Neapsi) da Faculdade de Educação da Unicamp.

Quadro 2. Relação de artigos autoeficacia e resiliência (2003-2008).

\begin{tabular}{lcl}
\hline Autoeficácia e resiliência & Frequência & Autores/ano de publicação \\
\hline Adolescência & 1 & Hamil (2003) \\
Desenvolvimento ao longo da vida & 2 & Jopp \& Rott (2006); Hammond (2004) \\
Recuperação pós-trauma & 0 & \\
Professores & 1 & Quing \& Day (2007) \\
Vida acadêmica & 2 & Martina \& Marsh (2008), Waxman Gray \& Padrón (2003) \\
Organizações & 1 & Stajkovic (2006) \\
Desafios de pesquisa & 1 & Luthar, Sawyer \& Brown (2006). \\
\hline
\end{tabular}

\section{Discussão}

\section{Autoeficácia e resiliência na adolescência}

Na adolescência, as crenças de autoeficácia tornam-se importantes para que o adolescente possa Tidar de forma bem-sucedida com as inúmeras mu具 danças que ocorrem nessa etapa de vida: as transfor出 mações púberes, o afloramento da sexualidade, a :D passagem do ambiente familiar para um ambiente imN pessoal, a complexidade do ensino médio, além da ocorrência de eventos de vida desvantajosos, como morte dos pais, doenças, divórcio, perdas financeiras

108 (Zimmerman, 2002).
Tais mudanças podem contribuir para que o adolescente se sinta sem controle pessoal, menos confiante em si mesmo, mais sujeito à avaliação social e menos automotivado, entrando muitas vezes, em depressão, envolvendo-se em atividades de risco, como consumo de drogas, atividade sexual prematura e comportamentos delinquentes, além de desengajamento das atividades sociais (Bandura, 1997). Falhas nos mecanismos autorregulatórios (por exemplo, a ausência de um pensamento antecipatório para estabelecer objetivos) podem levar a comportamentos disruptivos em sala de aula, à incapacidade de lidar com a própria saúde e à exposição à doença, em crianças e adolescentes (Zimmerman, 2002). 
A capacidade de deixar comportamentos aditivos, como deixar de beber, fortemente se associa à capacidade de autorregulação. A participação em um grupo de autoajuda pode favorecer o uso de múltiplas estratégias autorregulatórias que permitam o autodirecionamento e o engajamento do comportamento em relação à mudança desejada (Bandura, 2008). As habilidades autorregulatórias necessárias ao desenvolvimento de um senso de autoeficácia resiliente podem ser aprendidas em um programa de maestria que envolva capacidade de antecipar futuros estressores, reconhecimento de alternativas para lidar com eles, monitoramento da adequação das estratégias escolhidas e uso de autoincentivos capazes de suportar os esforços (Bandura, 1997).

Um exemplo de programa de maestria para adolescentes é o destinado a aumentar a resiliência para lidar com drogas, envolvendo professores, pais e outros interlocutores. Nesse programa, os adolescentes praticam regras e estratégias em situações simuladas, obtêm sucesso e são corrigidos em suas deficiências de forma suportiva e construtiva. Inclui, além de informações sobre os efeitos das drogas, habilidades de enfrentamento das pressões sociais, diminuição do uso de drogas e uso de estratégias que permitam aumentar o autoconceito como não usuário (Bandura, 1997).

A seguir, são apresentados alguns exemplos de estudos em que se investiga a resiliência em adolescentes, associando-a a crenças de autoeficácia. Hamill (2003), investigando resiliência em 46 adolescentes (média de 16 anos) em escola secundária de Nova York, refere-se à resiliência como processo dinâmico de adaptação positiva e desenvolvimento, apontando para a necessidade de definir termos como "competência" e "adversidade" quando se pretende estudar resiliência. A competência ou a ausência dela inclui autorrelatos e relatos dos pais com a descrição de comportamentos que denotem agressão, delinquência, inadequação social e acadêmica. A adversidade diz respeito à presença de estressores de uma destas condições: alto grau de adversidade (morte de pais, divórcio), acúmulo de estresse de moderada adversidade (problemas financeiros, mudança de escolas, brigas familiares) ou estressores crônicos (abuso de álcool por parte dos pais). Os achados apontam a presença de autoeficácia e mecanismos de enfrentamento em grupos competentes e resilientes, em contraposição à baixa competência e ao alto grau de adversidade em grupos mal-adaptados e em grupos com baixa competência e baixo grau de adversidade.

O estudo de Caprara, Pastorelli, Regalia, Scabini e Bandura (2005) investiga as relações entre a autoeficácia e a qualidade do funcionamento e a satisfação familiar. Os autores encontraram que, quanto mais os adolescentes percebem sua autoeficácia, mais eles apresentam uma comunicação aberta com seus pais, aceitam seu monitoramento em relação às atividades fora de casa e estão menos inclinados à discórdia e aos desentendimentos.

\section{Autoeficácia e resiliência no desenvolvimento ao longo da vida}

No campo do envelhecimento, dentro da perspectiva do desenvolvimento ao longo da vida (life-span), a resiliência é a capacidade de manutenção do desenvolvimento normal (não ótimo), a despeito da presença de ameaça e riscos (Staudinger et al., 1995). Segundo esses autores, a resiliência envolve os conceitos de plasticidade e capacidade de reserva. 0 primeiro diz respeito ao potencial de mudança do indivíduo e à sua flexibilidade para lidar com os desafios e as exigências, e está diretamente relacionado à capacidade de reserva do indivíduo, constituída por recursos internos (por exemplo, capacidade cognitiva e saúde física) e recursos externos (por exemplo, rede social e status econômico). Os recursos do indivíduo não são fixos e mudam ao longo do tempo. Dentro dessa perspectiva, a velhice é caracterizada como uma restrição geral da plasticidade e da capacidade de reserva, e o envelhecimento bem-sucedido relaciona-se com o equilíbrio resultante das perdas e dos ganhos. A resiliência teria como objetivo manter o funcionamento adaptativo ao longo do tempo.

Apesar de a velhice ter sido associada a perdas da capacidade física e cognitiva, os estudos têm demonstrado existir algumas compensações e ganhos: a capacidade de autorregulação permanece inalterada ao longo da vida por meio de fatores protetores do envelhecimento (Neri, 2006), fazendo com que determinadas áreas e mecanismos do self possam atuar como recursos para a resiliência e o crescimento, mantendo e 
otimizando o funcionamento da saúde, da inteligência e da memória (Bandura, 1997).

Neri (2006) aponta como mecanismos autorreguladores do self:a possibilidade de apoiar-se em diferentes papéis assumidos ao longo da vida; a substituição de metas de expansão por metas mais acomodativas; os mecanismos de comparação social com grupos semelhantes; a especialização cognitiva adquirida através da cultura e do trabalho, podendo significar o exercício de funções significativas, como o de supervisão, monitoria, aconselhamento (Fontes, 2006) e a seletividade socioemocional, ou seja, a capacidade de priorizar a rede social de contatos, dando preferência àqueles significativos, e de reduzir as emoções negativas.

No âmbito do envelhecimento, um estudo realizado com 51 idosos centenários - de 99 a 110 anos, em sua maioria mulheres, viúvas, 50\% deles institucionalizados, com saúde e cognição preservadas -, participantes do Heidelberg Centenarian Study, investigou quais os efeitos dos recursos básicos: cognição, saúde, rede social e extroversão, crenças de autoeficácia e atitude de otimismo como mediadores em relação à felicidade (Jopp \& Rott, 2006). Os resultados mostraram que os idosos centenários sentiam-se felizes, apesar das limitações físicas e cognitivas impostas pelo envelhecimento. Os recursos básicos (cognição, saúde e rede social) parecem não ter efeitos diretos sobre o bemestar. Já as crenças de autoeficácia e o otimismo contribuem para atenuar o impacto das perdas, atuando como recursos protetores do envelhecimento, ou seja, como fatores de resiliência.

Hammond (2004), através de um estudo qualitativo com 141 adultos da zona rural de Londres, com diversidade de experiências educacionais, investigou os efeitos da aprendizagem ao longo da vida sobre saúde, bem-estar, proteção, recuperação de dificuldades e capacidade de lidar potencialmente com situações estressoras, incluindo a doença crônica e a incapacidade. Os resultados demonstraram que a educação, ao longo da vida, permite que se desenvolvam a autoeficácia (crença na habilidade para controlar eventos significativos em sua vida). A autoestima; o propósito de vida; a esperança; a competência e a integração social, que promovem saúde mental e resiliência emocional diante da adversidade e das doenças.

\section{Autoeficácia e resiliência na recuperação pós-trauma}

As relações entre autoeficácia e resiliência são tidas como facilitadoras da recuperação pós-trauma. $O$ transtorno de estresse pós-traumático é definido pelo Diagnostic and Statistical Manual of Mental Disorders (DSM-IV) (1994) como a sintomatologia que se manifesta em indivíduos submetidos a eventos ou situações que envolvam ameaça de vida, como traumatização pós-guerra, desastres naturais (tornados, erupção de vulcões, tempestades, terremotos), ataques terroristas, traumatizações interpessoais (abuso sexual), roubos, assaltos, sequestros, viuvez.

Em geral, os modelos de pesquisa do Transtorno de Estresse Pós-Traumático (TEPT) focalizavam sua atenção nos resultados negativos da exposição a eventos traumáticos ou nos fatores que predispunham as pessoas às dificuldades para superação do trauma. Recentemente, a pesquisa tem enfatizado o estudo de características que protegem as pessoas de uma sequela física ou mental após exposição ao evento traumático, referindo-se à resiliência. Em amostra de veteranos do Vietnã, com ou sem estresse pós-traumático, a resiliência foi associada a baixos níveis de neuroticismo e psicotismo, altos níveis de lócus interno de controle e altos níveis de narrativas coerentes, indicando resolução de problemas, percepção do trauma como uma oportunidade de mudança, autodisciplina, senso de responsabilidade, otimismo, hardiness, senso de coerência, sabedoria e autoeficácia (Benight \& Bandura, 2004).

O modelo de agência proativa proposto por Bandura (1997; 2001) vem ao encontro dessa mudança de paradigma para explicar a recuperação pós-trauma, atribuindo aos mecanismos de autoeficácia a qualidade da vida emocional, a vulnerabilidade ao estresse e à depressão, a resiliência à adversidade e as escolhas do curso de vida. A autoeficácia atuaria como um mediador da recuperação pós-traumática.

A crença na capacidade de enfrentamento após a ocorrência de desastres naturais, como um terremoto, por exemplo, torna-se relevante para a aquisição de habilidades a serem desenvolvidas em programas de maestria, que deveriam incluir o desenvolvimento de habilidades como manutenção da vida diária; obtenção de recursos através da assistência social e do emprego; 
negociação do seguro com os órgãos federais; relacionamento com proprietários e contratos, para proteger a propriedade; recuperação das rotinas da vida e manutenção das relações familiares e sociais; e regulação das emoções de ansiedade, raiva e depressão (Benight \& Bandura, 2004).

\section{Autoeficácia e resiliência de professores}

A autoeficácia do professor está associada a importantes resultados educacionais, é um significante preditor da resiliência educacional e envolve: persistência diante das dificuldades, flexibilidade no uso de estratégias, manutenção do nível de desempenho acadêmico e modificação de formas de abordar tarefas, evitando, assim, o fracasso (Azzi, Polydoro \& Bzuneck, 2006; Bandura, 1997)

A autoeficácia atua também como um preditor do sucesso acadêmico do aluno, pois quanto maior a autoeficácia do professor, mais adequada será a escolha das estratégias de ensino, as explicações relativas ao conteúdo e as relativas a diferentes características dos alunos, o controle em sala de aula, as estratégias de enfrentamento e a persistência diante de situações difíceis, o entusiasmo, o compromisso e as metas pessoais (Azzi et al., 2006).

Quing e Day (2007), investigando a resiliência e a efetividade de 300 professores como fatores que contribuem para lidar com adversidades de diferentes fases da vida profissional e pessoal (início, meio e etapa final de carreira), analisam o impacto da autoeficácia, o senso de significado (sentido de vocação) e o suporte oferecido pela escola (treinamento, reconhecimento, promoção) como variáveis mediadoras. Os resultados mostraram que a resiliência variou de acordo com as etapas de vida profissional, e que a interação entre o senso de eficácia, a identidade profissional e o manejo dessa interação em determinados cenários é que determina o grau de resiliência do professor em relação aos obstáculos.

Maior resiliência foi encontrada quando o professor tinha um forte sentido de vocação ou missão, alto senso de autoeficácia e suporte oferecido pela escola. A autoeficácia e a resiliência, no início de carreira, são aumentadas em função do suporte oferecido pela administração e da oportunidade de carreira. A autoefi- cácia pode ser abalada quando os professores percebem que o sistema educacional vigente não está refletindo seus valores e interesses profissionais. O professor em fase final de carreira (26 anos) apresentava forte senso de autoeficácia diante das adversidades no trabalho e na vida pessoal. Ainda, a autoeficácia é aumentada em função de fatores como lideranças, staff, relação professores-alunos, comprometimento e eficácia percebida (Quing \& Day, 2007)

\section{Autoeficácia e resiliência acadêmica}

No âmbito educacional, Waxman et al. (2003) realizaram uma revisão dos estudos destinada a avaliar a resiliência de estudantes, ou seja, a capacidade de obter sucesso na escola, apesar da presença de condições adversas trazidas por características psicológicas, condições e experiência. São apontadas três categorias de estudo, dependendo do contexto específico onde o fenômeno é estudado: diferenças individuais na recuperação após trauma, resiliência em grupos de risco (envolvendo variáveis como pobreza, background familiar ou abuso), habilidades para adaptação, apesar das experiências estressantes (envolvendo variáveis como relações significativas, percepções da escola e envolvimento).

Apontado por Waxman et al. (2003), o Project Competence Studyé um estudo clássico cujo objetivo foi avaliar qual o impacto de eventos de vida estressante sobre os níveis de competência em uma amostra de 612 estudantes de escolas elementares, 200 deles acompanhados, juntamente com suas famílias, por mais de 10 anos. Os resultados mostraram que crianças com desvantagem apresentavam baixo coeficiente intelectual e nível socioeconômico, menos qualidades positivas na família, menor competência. No entanto, algumas delas eram competentes e não evidenciavam comportamentos destrutivos, o que suscitava o questionamento sobre o motivo de esses alunos, mesmo diante de condições adversas, não se tornarem disfuncionais no ambiente escolar. Esse fato demonstra que indivíduos resilientes interpretam diferentemente as experiências estressantes e o trauma, apontando para fatores do ambiente e para recursos do indivíduo e de resiliência.

Ainda segundo Waxman et al. (2003), estudantes resilientes percebem suas aulas muito mais favoráveis 
do que os não resilientes; têm autoconceito acadêmico e aspirações mais elevadas; percebem que os professores têm expectativas mais altas e utilizam mais feedback; percebem-se mais orientados para tarefas, ordem e organização; demonstram habilidades de liderança; trabalham bem em equipe em relação aos não resilientes. A falta de resiliência foi também associada às características do contexto instrucional: os estudantes trabalhavam de maneira passiva, com poucas atividades em grupo, tinham professores pouco questionadores, que forneciam poucas dicas e não os encorajavam a ajudar a si mesmos ou ao outro.

Da perspectiva do aluno, um currículo orientado para a maestria e para o projeto, aliado ao suporte, altas expectativas e o encorajamento ao engajamento e envolvimento são fortemente relacionados à resiliência. Da perspectiva do professor, a seleção, o treinamento e a manutenção dos professores envolvem o treinamento em habilidades de resiliência, como atenuar o impacto da exposição ao risco e desenvolver nos estudantes habilidades sociais, automonitoramento, autoavaliação e estratégias de autorreforçamento, além de habilidades de autorregulação relacionadas ao aumento da autoeficácia (Waxman et al. 2003).

Recentemente, Martina e Marsh (2008), ao estudarem os fatores preditores de resiliência acadêmica, fizeram distinção entre fatores distais - pais solteiros, etnia - e fatores proximais, que envolvem fatores escolares, de engajamento (participação em sala de aula, por exemplo), fatores relacionados aos pares e à família (suporte social) e fatores psicológicos, como autoeficácia, controle, senso de propósito e motivação.

\section{Autoeficácia e resiliência nas organizações}

O desempenho dos papéis profissionais dá-se em meio a pressões, percepções de ameaça e comparações sociais potencialmente limitantes. Nesse sentido, a crença na controlabilidade é fundamental para criar resiliência nas organizações. Os gerentes que acreditam que o meio é controlável buscam por melhores soluções (Bandura \&Wood, 1989). As crenças de autoeficácia podem ajudar a qualidade do funcionamento gerencial, de modo a torná-lo resiliente em relação a

112 estressores (Bandura, 1997).
Num experimento conduzido por Bandura e Wood (1989) para avaliar efeitos da controlabilidade sobre o desempenho organizacional, 40 homens e 20 mulheres de um programa de graduação na área de administração e negócios foram submetidos a uma tarefa de alocar empregados para diferentes funções, com base em critérios como habilidades, esforço requerido e características do empregado. Os resultados desse experimento indicaram que os gerentes que consideravam a organização como controlável mantinham um forte senso de autoeficácia, tendiam a metas desafiantes e exibiam um pensamento analítico efetivo. Além disso, quanto maior a experiência, mais o sistema de desempenho era regulado pela autoeficácia.

A manutenção das crenças de autoeficácia em ambientes organizacionais hostis envolve o desenvolvimento de habilidades destinadas a autoproteção. São elas: a análise de erros converter ameaças em oportunidades de mudança e minimizar as preocupações consigo próprio. A aquisição de tais habilidades reduz não apenas a vulnerabilidade aos efeitos dos erros sobre o desempenho, mas também o efeito das comparações sociais, principalmente em organizações bastante competitivas. O indivíduo deve ser levado a estabelecer comparações consigo próprio, com ênfase no autodesenvolvimento para aquisição de habilidades e na existência de altos e baixos, num contexto de aquisição de maestria para tarefas complexas (Bandura, 1997).

Um dos estudos encontrados na categoria organizacional associa autoeficácia e resiliência à motivação. Stajcovic (2006) apresenta o construto de "core de confiança", referindo-se a quatro variáveis: a imaginação do que e como pode ser feito (esperança), a crença de que se pode fazer tarefas específicas em determinados contextos (autoeficácia), a visualização positiva dos resultados (otimismo) e a crença de que se pode voltar atrás se as coisas não caminham bem (resiliência). O autor apoia-se em Bandura (1997) para definir autoeficácia, e para avaliar resiliência utiliza descrições como: "Eu posso voltar atrás quando estou errado"; "Eu não estou completamente recuperado emocionalmente e psicologicamente depois do acontecimento de 11 de setembro"; "Eu não lido bem com as coisas, sobre as quais eu não sei nada". Por meio dessas questões, pretende investigar a capacidade de adaptar-se aos estressores do ambiente organizacional. 


\section{Desafios de pesquisa}

As crenças de autoeficácia são tidas como uma variável bastante relevante, a ser mais bem investigada quando se pretende estudar resiliência (Luthar, Sawyer \&Brown, 2006).

Algumas necessidades são apontadas como relevantes na pesquisa sobre resiliência: a) distinguir fatores de proteção (algo que modifica numa direção positiva os efeitos do risco) de fatores de vulnerabilidade (aqueles que intensificam o risco); nem sempre o inverso de um fator protetor é um fator de vulnerabilidade; b) levar a efeito pesquisas que contemplem os aspectos genéticos e do meio ambiente; c) incluir avaliações do adulto pelos pares sobre seu desempenho como bons pais, esposos, chefes ou mentores e avaliações de crianças sobre o seu bem-estar e felicidade; d) ao escolher quais fatores de proteção ou de vulnerabilidade investigar, privilegiar aqueles que afetem grande número de pessoas, sejam suscetíveis de mudança, permaneçam durante longo tempo na vida das pessoas e atuem por meio de um efeito cascata, interferindo em outros (Luthar et al., 2006).

Pode-se dizer que as crenças de autoeficácia atendem aos quatro critérios sugeridos: afetam diferentes aspectos da vida, podem ser adquiridas em diferentes contextos ao longo da vida, seus resultados permanecem e atuam sobre diferentes aspectos relacionados à persistência diante da adversidade: emoções, depressão, escolhas de vida, habilidades e competências.

Hamill (2003), avaliando estratégias de enfrentamento em adolescentes como uma medida de resiliência, aponta a necessidade de utilizar, além de relatos verbais, também relatos de pares, pais e observações e registros de comportamento e competências, uma vez que os adolescentes podem indicar as estratégias adequadas, sem utilizá-las no cotidiano.

\section{Considerações Finais}

Neste artigo foram apresentados alguns estudos que discutem a relação "crenças de autoeficácia e resiliência", um campo de investigação que se configura como promissor, com imediata aplicabilidade de resultados, pois resistir às adversidades torna-se condição essencial para a qualidade de vida nas diferentes etapas do desenvolvimento, em um mundo em constante transformação, que aponta a necessidade de flexibilidade e acomodação.

Quando o foco é o desenvolvimento de crenças resilientes que protejam contra o desencorajamento e o enfrentamento de fracassos, ênfase especial deve ser dada à maestria em habilidades de enfrentamento: resolução de problemas, busca de suporte social, tomada de decisões e habilidades de comunicação. 0 desenvolvimento de experiências de domínio gera percepções de sucesso nas ações realizadas, contribuindo para fortalecer a crença de que se pode conviver com as contrariedades que os diversos ambientes proporcionam.

Outra consideração importante refere-se aos desafios de pesquisa para ampliação e aprofundamento da compreensão das relações aqui destacadas. Aspectos próprios do campo investigativo a serem mais bem trabalhados contemplam um amplo espectro, desde a necessidade de melhor definir os construtos utilizados, passando pela escolha de instrumentos de avaliação capazes de refletir as variáveis pesquisadas, bem como a relação entre elas, incluindo também delineamentos de estudos longitudinais.

As informações, os estudos e as considerações aqui feitas pretendem ter contribuído para destacar a importância dos constructos privilegiados, autoeficácia e resiliência. Certamente ainda há muito a ser debatido e investigado.

\section{Referências}

American Psychiatric Association. (1994). Diagnostic and statistical manual of mental disorders (4th ed). Washington, DC: Author. Retrieved September 29, 2010, from: <http:// www.psiqweb.med.br/site/?area=ES/Classificacoes \&palavra $=\&$ tipo $={ }^{\prime} \mathrm{D}^{\prime}>$.

Azzi, R. G., Polydoro, S. A. J., \& Bzuneck, J. A. (2006). Considerações sobre a autoeficácia docente. In R. G. Azzi \& S. A. J. Polydoro. Autoeficácia em diferentes contextos (pp.149-159). Campinas: Alínea.

Bandura, A. (1977). Self-efficacy: toward a unifying theory of behavioral change. Psychological Review, 84 (2), 191-215.

Bandura, A. (1986). Social foundations of thought and action: a social cognitive theory. Englewood Cliffs, NJ: PrenticeHall.

Bandura, A. (1997). Self-efficacy: the exercise of control. New York: W.H. Freeman. 
Bandura, A. (2001). Social cognitive theory: an agentic perspective. Annual Review of Psychology, 52, 1-26.

Bandura, A. (2002). Environmental sustainability by sociocognitive deceleration of population growth. In P. Schmuch \&W. Schultz (Eds.), The psychology of sustainable development (pp.209-238). Dordrecht: Kluwer.

Bandura, A. (2004). Swimming against the mainstream: the early years from chilly tributary to transformative mainstream. Behavior Research and Therapy, 42 (10), 613-630.

Bandura, A. (2006). Towards a psychology of human agency. Perspectives on Psychological Science. Retrieved April 28, 2006, from <http://www.des.emory.edu/mfp/BanPPS 2006>.

Bandura, A. (2008). A teoria social cognitiva na perspectiva da agência. In A. Bandura, R. G. Azzi \& S. Polydoro (Orgs.), Teoria social cognitiva: conceitos básicos (pp.69-95). Porto Alegre: Artmed.

Bandura A., \& Wood, R. (1989). Effect of perceived controllability and performancer standards on selfregulation of complex decision making. Journal of Personality and Social Psychology, 56 (5), 805-814.

Barreira, D. D., \& Nakamura, A. P. (2006). Resiliência e a autoeficácia percebida: articulação entre conceitos. Aletheia, 23, 75-80.

Benight C. C., \& Bandura, A. (2004). Social cognitive theory of posttraumatic recovery: the role of perceived selfefficacy. Behaviour Research and Therapy, 42 (10), 1129-1148. Retrieved March 20, 2007, from <http://www.science direct.com/science>.

Caprara, G. V., Pastorelli, C., Regalia, C., Scabini, E., \& Bandura, A. (2005). Impact of adolescents' filial self-efficacy on quality of family functioning and satisfaction. Journal of Research on Adolescence, 15 (1), 71-97

Fontes, A. P. (2006). Enfrentamento do estressenaidade adulta. Dissertação de mestrado não-publicada, Universidade Estadual de Campinas.

Hamill, S. K. (2003). Resilience and self-efficacy: the importance of efficacy beliefs and coping mechanisms in resilient adolescents. Colgate University Journal of the Sciences. Retrieved March 25, 2007, from <http://groups. colgate.edu/cjs/student_papers/2003/Hamill.pdf>.

Hammond, C. (2004). Impacts of life long learning upon of emotional resilience, psychological and mental health: fieldwork evidence. Oxford Review of Education, 30 (4), 551-568.
Jopp, D., \& Rott, C. (2006, June). Adaptation in very old age: exploring the role of resources, beliefs, and attitudes for centenarians happiness. Psychology and Aging, 21 (2), 266-280. Retrieved March 20, 2007, from <http://www. sciencedirect.com/science>

Lopes, E. S. L. (2005). Resiliência. In A. L. Neri (Org.), Palavraschave em gerontologia ( $2^{\mathrm{a}}$ ed., pp.178-181). Campinas: Alínea.

Luthar, S. S., Sawyer, J. A., \& Brown, P. J. (2006). Conceptual issues in studies of resilience: past, present, and future research. Annals of the New York Academy of Sciences, 1094 (pp.105-115). Retrieved March 25, 2007, from <http:// www.annalsnyas.org/cgi/content/full/1094/1/105>

Martina, A. J., \& Marsh, W. H. (2008). Academic buoyancy: towards an understanding of students' everyday academic resilience. Journal of School Psychology, 46 (1), 53-83. Retrieved January 26, 2009, from <http://www.science direct.com/science>

Neri, A. L. (2006). Teorias psicológicas do envelhecimento: Percurso histórico e teorias atuais. In E. V. Freitas, L. Py, F. A. X. Cançado, J. Doll \& M. L. Gorzoni. Tratado de geriatria e gerontologia (2a ed., pp.58-75). Rio de Janeiro: Guanabara Koogan.

Quing, G., \& Day, C. (November 2007). Teachers resilience: a necessary condition for effectiveness. Teaching and Teacher Education, 23 (8), 1302-1316. Retrieved March 15, 2008, from <http://www.sciencedirect.com/science>.

Stajkovic, A. D. (2006) Development of a core confidencehigher order construct. Journal of Applied Psychology, 91 (6), 1208-1224. Retrieved March 25, 2007, from <http:// web.mac.com>.

Staudinger, U. M., Marsiske, M., \& Baltes, P. B. (1995). Resiliência e níveis de capacidade de reserva na velhice: perspectivas da teoria do curso de vida. In A. L. Neri (Org.), Psicologia do envelhecimento (pp.195-228). Campinas: Papirus.

Waxman, H. C., Gray, J. P., \& Padrón, Y. N. (2003). Review of research on educational resilience. Center for Research on Education, Diversity \& Excellence. Retrieved March 25, 2007, from <http://www.cal.org/crede/pubs/research/RR11.pdf>.

Zimmerman, B. J. (2002). Achieving self-regulation. The trial and triumph of adolescence. In F. Pajares \& T. Urdan (Eds.), Academic motivation of adolescence (Vol. 2). Greenwich, CT: Information Age Publishing.

Recebido em: 21/5/2009

Versão final reapresentada em: 7/10/2011

Aprovado em: 3/11/2011 